\title{
Structural Basis of Conserved Flagellin-mediated TLR5 Stimulation
}

\author{
Wan Seok Song ${ }^{1}$, Ye Ji Jeon ${ }^{1}$, Byeol Namgung ${ }^{1}$, Minsun Hong² ${ }^{\star}$, and Sung-il Yoon ${ }^{1^{*}}$ \\ ${ }^{1}$ Division of Biomedical Convergence, College of Biomedical Science, Kangwon National University, \\ Chuncheon 24341, Republic of Korea ${ }^{2}$ Division of Biomedical Science and Technology, Yonsei University, \\ Wonju 26493, Republic of Korea
}

The bacterial flagellum is a whip-like organelle, which extends from the cell membrane to the extracellular space and is involved in motility. The flagellar filament of bacteria assembles through the helical selfpolymerization of flagellin protomers. In the host, flagellin is detected as a common molecular pattern of diverse bacteria by Toll-like receptor 5 (TLR5) and activates innate immune response. Here, we report that TLR5 interacts with various flagellins from the Gammaproteobacteria and Firmicutes groups with comparable binding affinity and, as a result, is activated by flagellins in similar potency. To reveal the structural mechanism used by TLR5 to recognize various flagellins, we have determined the crystal structure of a complex between TLR5 and flagellin. In the complex structure, TLR5 accommodates highly conserved flagellin residues using a cavity that is generated by the LRR9 loop of TLR5. Furthermore, our mutational study on flagellin showed that an arginine residue and its neighboring glutamate residue of flagellin play a key role in TLR5 interaction and activation. These results provide a structural basis of the conserved flagellin-mediated TLR5 activation mechanism. 